\title{
Standardizing Terminology and Assessment for Orofacial Conditions in Juvenile Idiopathic Arthritis: International, Multidisciplinary Consensus-based Recommendations
}

\author{
Peter Stoustrup ${ }^{\circledR}$, Cory M. Resnick, Thomas Klit Pedersen, Shelly Abramowicz, \\ Ambra Michelotti, Annelise Küseler, Carlalberta Verna, Christian J. Kellenberger, \\ Ellen Berit Nordal ${ }^{(\mathbb{D})}$, Giuliana Caserta ${ }^{(\mathbb{D}}$, Iveta Jankovska, Josefine Mareile Halbig (1), \\ Kasper Dahl Kristensen, Linda Z. Arvidsson, Lynn Spiegel, Mathew L. Stoll, Melissa Lerman, \\ Mia Glerup, Patrizia Defabianis, Paula Frid, Per Alstergren, Randy Q. Cron, Sarah Ringold, \\ Sven Erik Nørholt, Timo Peltomäki, Troels Herlin, Zachary S. Peacock, and Marinka Twilt, \\ and the TMJ Juvenile Arthritis Working Group
}

\begin{abstract}
Objective. To propose multidisciplinary, consensus-based, standardization of operational terminology and method of assessment for temporomandibular joint (TMJ) involvement in juvenile idiopathic arthritis (JIA).

Methods. Using a sequential expert group-defined terminology and methods-of-assessment approach by (1) establishment of task force, (2) item generation, (3) working group consensus, (4) external expert content validity testing, and (5) multidisciplinary group of experts final Delphi survey consensus.

Results. Seven standardized operational terms were defined: TMJ arthritis, TMJ involvement, TMJ arthritis management, dentofacial deformity, TMJ deformity, TMJ symptoms, and TMJ dysfunction. Conclusion. Definition of 7 operational standardized terms provides an optimal platform for communication across healthcare providers involved in JIA-TMJ arthritis management. (First Release January 15 2019; J Rheumatol 2019;46:518-22; doi:10.3899/jrheum.180785)
\end{abstract}

Key Indexing Terms:

JUVENILE IDIOPATHIC ARTHRITIS TEMPOROMANDIBULAR JOINT CHILDHOOD

From the Department of Dentistry and Oral Health, Aarhus University, Department of Oral and Maxillofacial Surgery, Aarhus University Hospital; Department of Orthodontics, Aarhus University; Department of Pediatrics, Aarhus University Hospital; Pediatric Rheumatology Clinic, Pediatrics and Adolescent Medicine, Aarhus University Hospital; Section of Oral Surgery and Oral Pathology, Aarhus University, Aarhus, Denmark; Harvard School of Dental Medicine and Harvard Medical School; Department of Plastic and Oral Surgery, Boston Children's Hospital; Department of Oral and Maxillofacial Surgery, Massachusetts General Hospital, Boston, Massachusetts; Oral and Maxillofacial Surgery and Pediatrics, Emory University School of Medicine, Children's Healthcare of Atlanta, Atlanta, Georgia; University of Alabama at Birmingham, Birmingham, Alabama; Perelman School of Medicine, University of Pennsylvania; Children's Hospital of Philadelphia, Philadelphia, Pennsylvania; Seattle Children's Hospital, Seattle, Washington, USA; Department of Neuroscience, Reproductive Sciences and Oral Sciences, Section of Orthodontics, University of Naples Federica II, Naples; Dental School, Section of Paediatric Dentistry, University of Turin, Turin, Italy; Department of Orthodontics and Pediatric Dentistry, UZB, University Center for Dental Medicine, Basel; Department of Diagnostic Imaging, University Children's Hospital Zurich, Switzerland; Department of Pediatrics, University of North Norway and Department of Clinical Medicine, UiT the Arctic University of Norway, Tromso; Section of Orthodontics, Department of Maxillofacial Radiology, Institute of Clinical Dentistry, University of Oslo, Oslo; Department of Otorhinolaryngology, Division of Oral and Maxillofacial Surgery, University Hospital North Norway; The Public Dental Service Competence Centre of North Norway; Department of Clinical Medicine, Faculty of Health Sciences, The Arctic
University of Norway, Tromso, Norway; Department of Orthodontics, Faculty of Dentistry, Rigas Stradins University, Riga, Latvia; Department of Pediatrics, Division of Rheumatology, The Hospital for Sick Children, University of Toronto, Toronto, Ontario; Department of Pediatrics, Cumming School of Medicine, University of Calgary; Alberta Children's Hospital, Calgary, Alberta, Canada; Malmo University, Faculty of Odontology, Orofacial Pain Unit, Malmo and Skane University Hospital, Specialized Pain Rehabilitation, Lund, Sweden; Department of Ear and Oral Diseases, Tampere University Hospital; Faculty of Medicine and Life Sciences, University of Tampere; Institute of Dentistry and University of Eastern Finland, Kuopio, Finland.

P. Stoustrup, Associate Professor, Section of Orthodontics, Department of Dentistry and Oral Health, Aarhus University; C.M. Resnick, Assistant Professor, Oral and Maxillofacial Surgery, Harvard School of Dental Medicine and Harvard Medical School, and Department of Plastic and Oral Surgery, Boston Children's Hospital; T.K. Pedersen, PhD, Consultant Orthodontist, Professor, Department of Oral and Maxillofacial Surgery, Aarhus University Hospital, Section of Orthodontics, Aarhus University; S. Abramowicz, DMD, MPH, FACS, Associate Professor, Oral and Maxillofacial Surgery and Pediatrics, Emory University School of Medicine, Children's Healthcare of Atlanta; A. Michelotti, DDS, Orthodontist, Department of Neuroscience, Reproductive Sciences and Oral Sciences, Section of Orthodontics, University of Naples Federica II; A. Küseler, DDS, PhD, Department of Orthodontics, Aarhus University Hospital; C. Verna, DDS, PhD, Professor, Head, Department of Orthodontics and Pediatric Dentistry, UZB, University Center for Dental Medicine; C.J. Kellenberger, MD, Prof., Dr. med., Department of Diagnostic Imaging, University Children's Hospital Zurich; E. Berit 
Temporomandibular joint (TMJ) arthritis is common in juvenile idiopathic arthritis (JIA) ${ }^{1,2}$. TMJ arthritis may impair joint mobility and masticatory function, cause TMJ degeneration, lead to reduced dentofacial growth, create orofacial pain, and affect general quality of life $\mathrm{e}^{3,4,5,6,7,8}$. Treatment is complex and multidisciplinary, involving pediatric rheumatologists, maxillofacial surgeons, orthodontists, radiologists, pediatric dentists, occupational and physiotherapists, and orofacial pain specialists $^{9}$.

Research in TMJ arthritis has increased exponentially over the last decade. As this field has grown, so has confusion over terminology. Recent systematic reviews have highlighted the need for a standardized set of JIA-associated TMJ arthritis definitions ${ }^{6,7,8}$. Standardization is critical to enhance research comparability and care provider communication.

The objectives of our study were to use a consensus-based approach to propose the following: (1) a standardized terminology for JIA-associated TMJ arthritis; and (2) methods of assessment of TMJ arthritis in patients with JIA.

Nordal, MD, PhD, Department of Pediatrics, University of North Norway, and Department of Clinical Medicine, UiT the Arctic University of Norway; G. Caserta, DDS, Lecturer, CIR - Dental School, Section of Paediatric Dentistry, University of Turin; I. Jankovska, DDS, PhD, Assistant Professor, Department of Orthodontics, Faculty of Dentistry, Rigas Stradins University; J.M. Halbig, Dr. med. Dent., DDS, The Public Dental Service Competence Centre of North Norway; K.D. Kristensen, DDS, PhD, Guest Researcher, Section of Orthodontics, Department of Dentistry and Oral Health, Aarhus University; L.Z. Arvidsson, DDS, PhD, Associate Professor, Department of Maxillofacial Radiology, Institute of Clinical Dentistry, University of Oslo; L. Spiegel, MD, FRCPC,

Department of Pediatrics, Division of Rheumatology, The Hospital for Sick Children, University of Toronto; M.L. Stoll, MD, PhD, MSCS,

University of Alabama at Birmingham; M. Lerman, MD, MSc, PhD, Assistant Professor of Clinical Pediatrics, Perelman School of Medicine, University of Pennsylvania, and Children's Hospital of Philadelphia; M. Glerup, MD, Department of Pediatrics, Aarhus University Hospital; P. Defabianis, MD, DDS, Associate Professor, Chairman and Program Director of Pediatric Dentistry, Section of Pediatric Dentistry, CIR-Dental School, University of Turin; P. Frid, DDS, Department of Otorhino-laryngology, Division of Oral and Maxillofacial Surgery, University Hospital North Norway, and Public Dental Service Competence Centre of North Norway, and Department of Clinical Medicine, Faculty of Health Sciences, The Arctic University of Norway; P. Alstergren, DDS, PhD, Malmo University, Faculty of Odontology, Orofacial Pain Unit, and Skane University Hospital, Specialized Pain Rehabilitation; R.Q. Cron, MD, $P h D$, University of Alabama at Birmingham; S. Ringold, MD, MS, Seattle Children's Hospital; S.E. N $\phi$ rholt, DDS, PhD, Clinical Professor, Consultant Surgeon, Department of Oral and Maxillofacial Surgery, Aarhus University Hospital, and Section of Oral Surgery and Oral Pathology, Aarhus University; T. Peltomäki, DDS, PhD, Department of Ear and Oral Diseases, Tampere University Hospital, and Faculty of Medicine and Life Sciences, University of Tampere, and Institute of Dentistry and University of Eastern Finland; T. Herlin, MD, DMSci, Pediatric Rheumatology Clinic, Pediatrics and Adolescent Medicine, Aarhus University Hospital; Z.S. Peacock, DMD, MD, Assistant Professor, Department of Oral and Maxillofacial Surgery, Massachusetts General Hospital, and Harvard School of Dental Medicine; M. Twilt, MD, MSCE, PhD, Assistant Professor, Department of Pediatrics, Cumming School of Medicine, University of Calgary, and Alberta Children's Hospital.

Address correspondence to Dr. P. Stoustrup, Section of Orthodontics, Department of Dentistry and Oral Health, Aarhus University, Vennelyst Boulevard 9-11,8000 Aarhus C, Denmark.E-mail: pstoustrup@dent.au.dk Accepted for publication September 24, 2018.

\section{MATERIALS AND METHODS}

This study was conducted using a series of sequential iterations including the following: (1) establishment of task force and item generation; (2) working group consensus meeting and drafting of provisional recommendations; (3) content validity testing of provisional recommendations by external experts; and (4) Delphi survey to reach final consensus (Figure 1).

Task force assembly, item generation, consensus meeting. The task force was assembled with members of the TMJ Juvenile Arthritis Working group (TMJaw; formerly known as euroTMjoint research network), an international, multidisciplinary, open group studying TMJ arthritis in JIA, and includes researchers from all specialties involved in JIA-related TMJ arthritis management. Members of the terminology task force were identified based on clinical experience and scientific contributions. The task force included 1 pediatric rheumatologist (MT), 2 oral maxillofacial surgeons (SA, CR), and 2 orthodontists (TKP, PS), and represents 1 European and 3 North American centers.

For item generation, the task force identified TMJ arthritis-related terms used in existing JIA literature ${ }^{7,8}$. In February 2017, those terms were distributed to all members of TMJaw by e-mail, with an invitation to par-

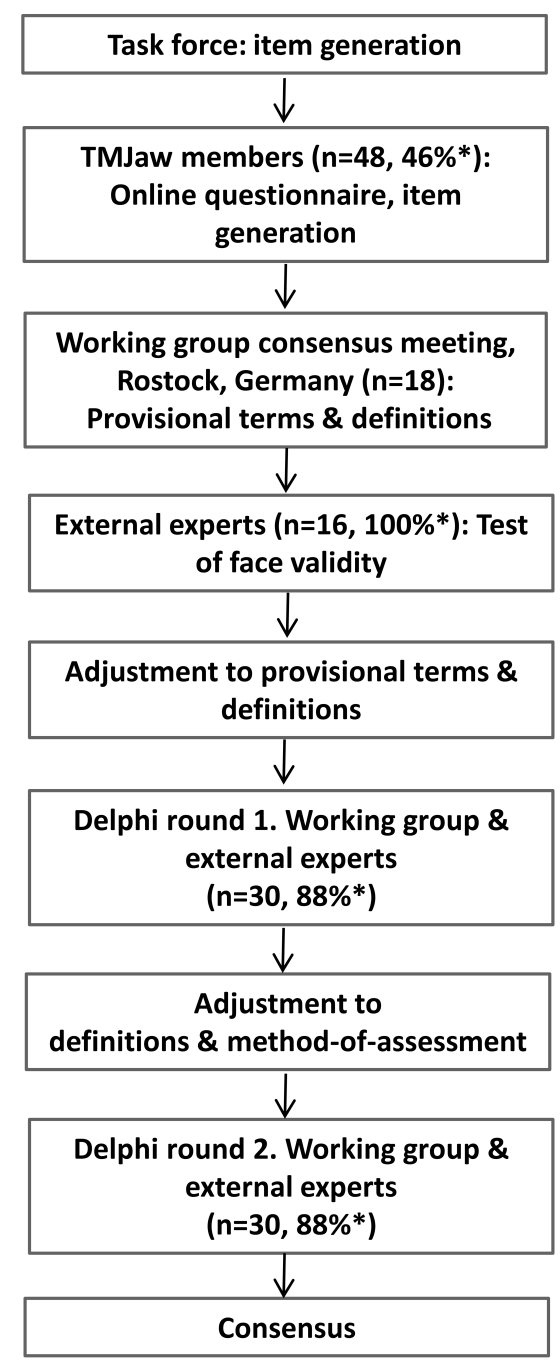

Figure 1. Flowchart of consensus process. * Response rate. TMJaw: Temporomandibular joint Juvenile Arthritis Working Group. 
ticipate in an online questionnaire. The participants were asked to report their understanding of these terms and the role of different specialties involved in TMJ arthritis management.

Based on the results of the online questionnaire, the terminology task force generated 6 provisional terms for discussion at the TMJaw meeting in Rostock, Germany, in March 2017. The provisional terms were adjusted and definitions for each term were established based on consensus from the group.

Test of face validity. In April 2017, 16 external experts were invited to assess the validity of the provisional terms and definitions generated at the Rostock consensus meeting. The external experts were identified based on TMJ arthritis clinical expertise and scientific merit. The external experts were asked to assess validity, suggest improvements, and address redundancies for the provisional terms. The task force then adjusted the provisional terms and definitions accordingly.

Delphi survey. In September 2017, participants from the Rostock consensus meeting $(n=18)$ and external experts $(n=16)$ were invited to participate in an online Delphi survey to assess agreement with the provisional terms. Participants were asked to respond to each term and definition with "agree," "agree with minor changes," or "do not agree." Participants were also asked to suggest improvements to the terms and definitions, and to define methods of assessment for each term from the following options: (1) contrast-enhanced magnetic resonance imaging (MRI); (2) MRI without contrast; (3) 3-D imaging [e.g., computed tomography (CT) or cone-beam CT]; (4) conventional radiology (e.g., cephalograms and panoramic radiographs); (5) ultrasonography; (6) clinical examination; (7) patient-reported outcomes; and (8) other (e.g., 3-D photographs and scintigraphy). Participants could select multiple options.

The provisional terms and definitions were adjusted based on the results of this Delphi survey. These results were provided to participants before initiation of the next iteration in December 2017. In this final Delphi round, participants could "agree" or "disagree" with each term and definition. Only terms and definitions that received "agreement" by $>80 \%$ of participants were included in the final recommendations. The results were summarized, and final consensus was reached in February 2018.

\section{RESULTS}

Thirty experts participated in the final Delphi survey (Figure 1). The following specialties were represented: pediatric rheumatology $(n=10)$, maxillofacial surgery $(n=5)$, orthodontics $(n=8)$, pediatric dentistry $(n=3)$, radiology $(n=3)$, and orofacial pain $(n=1)$. Based on results of the first round of the Delphi survey, the number of terms was expanded from 6 to 7; the term TMJ disability was divided into TMJ symptoms and TMJ dysfunction. All 7 provisional terms received a Delphi survey agreement score $>80 \%$ (Table 1 ).
TMJ arthritis. Arthritis is defined as "inflammation in a joint." The term TMJ arthritis, therefore, is intended to indicate the presence of active TMJ inflammation, and is independent of signs and symptoms; hence it can be present with or without any signs or symptoms. To add the qualifier "active" to the definition was considered redundant by some, but this word was ultimately included to highlight the strict characteristics of the term, which refers only to inflammation and not joint damage per se. The term chronic TMJ arthritis has been used in literature, but the task force recommends avoiding it because of imprecision, and chronic but not active TMJ arthritis falls under the term TMJ involvement.

Contrast-enhanced MRI is the current method for assessment of active inflammation in TMJ arthritis ${ }^{7}$. Other methods (e.g., clinical examination and patient reporting) may suggest the presence of TMJ arthritis but cannot confirm the presence of inflammation. Contrast-enhanced MRI was the only method of assessment that received a recommendation score $>80 \%$. A recently published MRI scoring system is recommended ${ }^{10,11}$.

TMJ involvement and TMJ arthritis management. TMJ involvement is defined as "abnormalities presumed to be the result of TMJ arthritis." This term is less restrictive than TMJ arthritis. The presence of active TMJ inflammation (TMJ arthritis) is not a prerequisite for TMJ involvement, but TMJ arthritis implies the presence of TMJ involvement. The term TMJ involvement is intended for the following: (1) clinical situations in which no contrast-enhanced MRI verification of active TMJ inflammation has occurred but where signs, symptoms, and/or radiological findings suggest the presence of actual or former TMJ arthritis; (2) patients with no current MRI evidence of active TMJ inflammation (TMJ arthritis) but with abnormalities indicating previous TMJ arthritis. Once the TMJ has been inflamed, it is prospectively considered "involved" regardless of the current inflammatory state.

By definition, TMJ arthritis management embraces diagnosis, treatment, and monitoring of TMJ arthritis and involvement. Methods of assessment include contrast-enhanced MRI, 3-D imaging, clinical examination, and patient-reported outcomes.

Table 1. Recommended standardized operational terminology, definitions, and agreement with definitions in patients with JIA.

\begin{tabular}{ll}
\hline Terms & Definitions \\
\hline TMJ arthritis & Active inflammation in the TMJ \\
TMJ involvement & Abnormalities presumed to be the result of TMJ arthritis \\
TMJ arthritis management & Diagnosis, treatment, and monitoring of TMJ arthritis and involvement \\
Dentofacial deformity & Abnormality in growth, development, structure, and/or alignment of the facial bones and dentition \\
TMJ deformity & Abnormality in growth, development, or structure of the osseous and/or soft-tissue components \\
& of the TMJ \\
TMJ symptoms & Patient- or parent-reported conditions related to TMJ arthritis or involvement \\
TMJ dysfunction & Physician-reported functional examination abnormalities related to TMJ arthritis or involvement
\end{tabular}

The terminology applies to subjects with a diagnosis of JIA. JIA: juvenile idiopathic arthritis; TMJ: temporomandibular joint.

Personal non-commercial use only. The Journal of Rheumatology Copyright $\odot$ (2019. All rights reserved 
Table 2. Standardized terminology and method of assessment in patients with juvenile idiopathic arthritis.

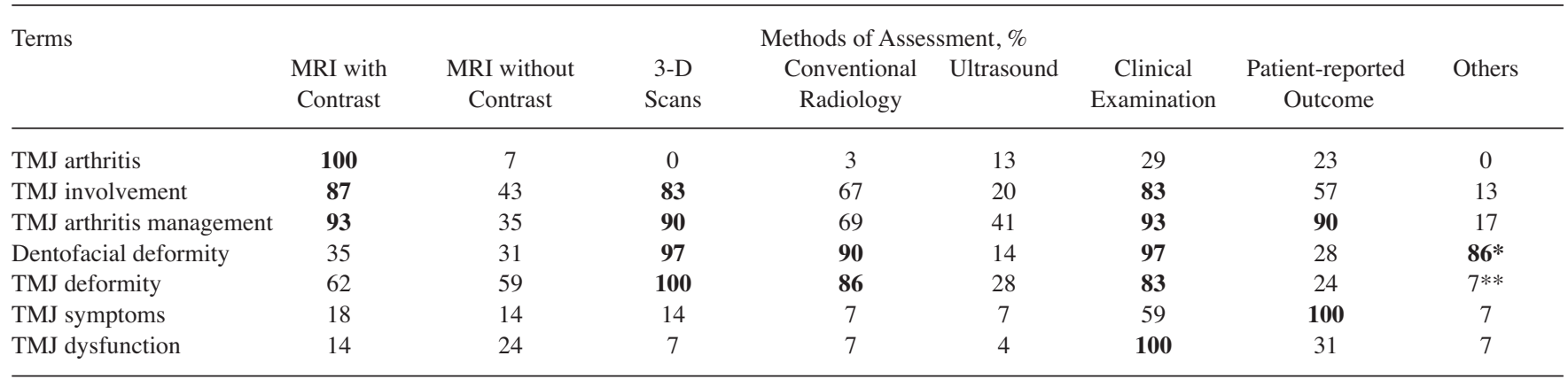

Values in bold face represent the method of assessment receiving and agreement score $\geq 80 \%$. Specific methods of assessment are recommended when expert assessment $\geq 80 \%$. Methods of assessment with a recommendation score $<80 \%$ should not be discounted because they may still be important in special situations. * "Others" represents 3-D photography and clinical photos. ** Scintigraphy was included as an option to assess TMJ deformity in the second Delphi round and was recommended by $28 \%$ of the participants. TMJ: temporomandibular joint; MRI: magnetic resonance imaging.

Dentofacial deformity and TMJ deformity. Dentofacial deformity refers to growth deviation that occurs as a result of TMJ arthritis in patients with JIA. Growth deviation may affect the morphology and position of the mandible, maxilla, and/or dental occlusion ${ }^{12,13}$. The recommended assessment of dentofacial deformity includes 3-D imaging, conventional radiography, clinical examination, and photographs. Recent recommendations are available ${ }^{13}$.

TMJ deformity indicates arthritis-related alteration of the anatomy of the TMJ. This term does not cover signs and symptoms (which are considered in another term, TMJ dysfunction), but rather is limited to anatomic deformities. Conventional radiographs and 3-D imaging are used to assess osseous TMJ deformities, with the caveat that soft tissue changes, which are often present in TMJ deformities, will not be visible with these imaging techniques but would require MRI evaluation.

TMJ symptoms and TMJ dysfunction. TMJ symptoms refers to patient/parent-reported measures. In contrast, the term TMJ dysfunction addresses clinical examination signs of abnormal mandibular function believed to be related to TMJ involvement. Recent recommendations for clinical orofacial examination in JIA are available ${ }^{8}$. The final recommendations for standardized terminology and definitions are presented in Table 1. The final recommendations for methods of assessment are shown in Table 2.

\section{DISCUSSION}

In our study, the TMJaw group has described and defined the most common terms used in TMJ arthritis research and is uniquely positioned to provide these recommendations for standardization of terminology representing multiple specialties and many North American and European TMJ arthritis research centers. Consensus-based standardization of terminology provides an optimal platform for communication across healthcare providers involved in research and management of TMJ arthritis in JIA. An important qualifier is that these terms apply only to patients with JIA. It must be noted that TMJ symptoms and/or TMJ dysfunction may not be directly attributable to JIA, as in a patient with myofascial pain disorder. To date, no reliable diagnostic method exists to distinguish between JIA-related orofacial symptoms/ dysfunction and similar findings due to other etiologies. This project did not intend to define terms applicable to other temporomandibular dysfunction; further studies are required.

Additionally, the methods of assessment that received recommendation scores $>80 \%$ represent the most frequent diagnostic measures used to assess each term and serve as a guide. Methods of assessment with a recommendation score $<80 \%$ should not be discounted, however, because they may still be useful in special clinical scenarios.

The unequal numbers of participating experts representing different specialties may be a limitation to our study; however, we are convinced that it strengthens the recommendations that so many different experts agree on the proposed standardized terminology.

We encourage investigators and clinicians to use the recommended terms and definitions in future publications as a reference standard. We suggest including the following text: "terminology adheres to JIA-TMJaw consensus-based standardized terminology."

\section{ACKNOWLEDGMENT}

We acknowledge Drs. Bernd Koos, Rotraud Saurenman, Tore A. Larheim, Nikolay Tzaribachev, Severine Cuillaume-Czitrom, and Zane Krisjane for their contribution to this project.

\section{REFERENCES}

1. Stoll ML, Sharpe T, Beukelman T, Good J, Young D, Cron RQ. Risk factors for temporomandibular joint arthritis in children with juvenile idiopathic arthritis. J Rheumatol 2012;39:1880-7.

2. Weiss PF, Arabshahi B, Johnson A, Bilaniuk LT, Zarnow D, Cahill AM, et al. High prevalence of temporomandibular joint arthritis at disease onset in children with juvenile idiopathic arthritis, as detected by magnetic resonance imaging but not by ultrasound. Arthritis Rheum 2008;58:1189-96. 
3. Arvidsson LZ, Fjeld MG, Smith HJ, Flato B, Ogaard B, Larheim TA. Craniofacial growth disturbance is related to temporomandibular joint abnormality in patients with juvenile idiopathic arthritis, but normal facial profile was also found at the 27-year follow-up. Scan J Rheumatol 2010;39:373-9.

4. Arvidsson LZ, Flato B, Larheim TA. Radiographic TMJ abnormalities in patients with juvenile idiopathic arthritis followed for 27 years. Oral Surg Oral Med Oral Pathol Oral Radiol Endod 2009;108:114-23.

5. Fjeld MG, Arvidsson LZ, Smith HJ, Flato B, Ogaard B, Larheim TA. Relationship between disease course in the temporomandibular joints and mandibular growth rotation in patients with juvenile idiopathic arthritis followed from childhood to adulthood. Pediatr Rheumatol Online 2010;8:13.

6. Frid P, Nordal E, Bovis F, Giancane G, Larheim TA, Rygg M, et al; Paediatric Rheumatology International Trials Organisation.

Temporomandibular joint involvement in association with quality of life, disability, and high disease activity in juvenile idiopathic arthritis. Arthritis Care Res 2017;69:677-86.

7. Kristensen KD, Stoustrup P, Küseler A, Pedersen TK, Twilt M, Herlin T. Clinical predictors of temporomandibular joint arthritis in juvenile idiopathic arthritis: a systematic literature review. Semin Arthritis Rheum 2016;45:717-32.
8. Stoustrup P, Twilt M, Spiegel L, Kristensen KD, Koos B, Pedersen TK, et al; euroTMjoint Research Network. Clinical orofacial examination in juvenile idiopathic arthritis: international consensus-based recommendations for monitoring patients in clinical practice and research studies. J Rheumatol 2017;44:326-33.

9. Øgaard B. Introduction. Semin Orthodont 2015;21:71.

10. Kellenberger CJ, Junhasavasdikul T, Tolend M, Doria AS. Temporomandibular joint atlas for detection and grading of juvenile idiopathic arthritis involvement by magnetic resonance imaging. Pediatr Radiol 2018;48:411-26.

11. Tolend MA, Twilt M, Cron RQ, Tzaribachev N, Guleria S, von Kalle T, et al. Towards establishing a standardized magnetic resonance imaging scoring system for temporomandibular joints in juvenile idiopathic arthritis. Arthritis Care Res 2018:70:758-67.

12. Chatzigianni A, Kyprianou C, Papadopoulos MA, Sidiropoulou S. Dentoalveolar characteristics in children with juvenile idiopathic arthritis. J Orofac Orthop 2018;79:133-9.

13. Stoustrup P, Iversen CK, Kristensen KD, Resnick CM, Verna C, Norholt SE, et al. Assessment of dentofacial growth deviation in juvenile idiopathic arthritis: reliability and validity of three-dimensional morphometric measures. PLoS One 2018;13:e0194177. 